\title{
Malaria from Africa blows hot and cold
}

\begin{abstract}
Although the spread of malaria is still not controlled, new findings show that immunity to severe forms of malaria is acquired after only one or two infections and may depend on well-conserved antigens (pages 340-343).
\end{abstract}

IN A RESEARCH area in which bad news is more frequent than good, the report from Newbold's group on page 340 of this issue $^{1}$ brings new hope that mortality from severe malaria caused by Plasmodium falciparum can be controlled. Their analysis of longitudinal clinical data obtained in various African regions with differing transmission intensities shows that resistance to severe disease is acquired very rapidly. This suggests that target antigens are well-conserved and, therefore, that vaccination could be an easier task to achieve than previously thought.

This good news is welcome: with an estimated 2.5 billion individuals at risk, about 1 billion carrying parasites, 2-3 million deaths per year and a research investment 1,000 to 10,000 times less per case than that for AIDS, malaria seems both a major threat to humans living in developing countries and, paradoxically, one of today's most neglected diseases. The recent evidence that resistance of malaria to chloroquine, the main compound used for primary health care, could raise those mortality rates as much as 8 times $^{2,3}$, and that drug resistance extends to all compounds, obviously darkens an already very gloomy picture.

The study by Newbold and colleagues ${ }^{1}$ illustrates the welcome trend in malaria research in which the interactions between the malaria parasite and the human host are examined as closely as the molecular aspects of the parasite have been examined in the past. The investigators addressed the question of how quickly immunity develops to the most severe forms of malaria, that is, those responsible for the high death toll. For this, they relied on longitudinal clinical records in four areas of Africa that, because of climatic differences, differed in malaria incidence rates. They also used one very simple, though objective, criterion to define severe malaria: hospitalization. Severe forms of malaria are normally confined to children less than 5 years of

\section{Pierre Druilhe \& JEAN LOUIS PÉRIGNON}

age in holoendemic areas of Africa. However, the study predicts that resistance to severe disease can be established after as few as one or two infections, which is earlier than previously thought.

'Complex' is probably the only word that all malariologists would agree on to describe host-parasite interactions. Only asexual erythrocytic forms of the parasite are pathogenic; however, in highly endemic regions, they cause a wide range of clinical outcomes, from death within 24 hours in younger children (and nonindigenous travellers), to well-tolerated and asymptomatic massive parasite loads

elicited by each type of disease, and that they rely on distinct mechanisms directed against different antigens. It is now agreed that the antigens of the sporozoite stage (injected by the mosquito; see Fig. 2), the intra-hepatic schizont (clinically silent), the asexual erythrocytic schizonts, the merozoites released by bursting red cells and the sexual stage gametocytes are all different targets. Clinical observations have shown that anti-parasitic immunity (whether strain-specific or strain-independent, that is, directed against conserved targets) and anti-disease immunity are different. In the latter, immunity to mild malaria (the ability to withstand massive parasite loads) would have now to be distinguished from immunity to the severest forms (of which cerebral malaria would possibly constitute a separate group, as indicated in the Newbold study by the delay in developing resistance).

Will a reduction in transmission be deleterious? By demonstrating that resistance to severe malaria develops faster in areas of high transmission, the Newbold study is in keeping with recent reports showing that the incidence of severe disease is by no means in direct proportion to the rate of transmission, but instead the reverse is true $e^{4,5}$. This contributes to the dismantling of the long-held view that decreasing the rate of transmission must be somehow helpful and beneficial to exposed populations. It questions the validity of current control strategies, particularly those relying on insecti-

in older children $\left(10^{9}\right.$ to $10^{11}$ parasites produce a true parasitic septicemia), up to lifelong cohabitation with hardly detectable parasite loads in adults (see Fig. 1). Death is not caused by cerebral malaria alone, but can be the outcome of other serious manifestations of the disease, such as severe anemia, pulmonary and kidney complications, metabolic acidosis or hypoglycemia. It is now better understood that different types of immunity are cide-impregnated bednets to reduce man-mosquito contact (at least in hightransmission areas; the benefit obviously remains in low-transmission regions).

These concerns are counterbalanced by good news for vaccine development. A chief implication of the study is that polymorphic antigens or variant antigens are very unlikely to be the target of the immune responses protecting against severe malaria. This concept goes against 


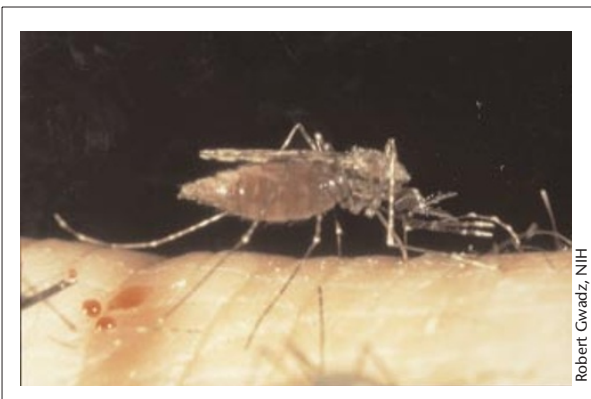

Human infection is initiated by the bite of a mosquito (Anopheles gambiae is shown here), which introduces infectious sporozoites into the circulatory system.

the tide of current opinion, as the identification of polymorphic regions in many malarial antigens and the recent discovery of the large family of var genes (a series of molecules expressing different antigenic motifs) have driven many groups to focus on those antigens as vaccine candidates.
That protection can be conferred by conserved antigens is certainly a reason to be more optimistic for the future ${ }^{6}$.

When the WHO/UNDP/WB special program was launched in 1974, the goal was to develop ' $a$ ' malaria vaccine, as it was implicit that a single one should be able to prevent all manifestations. In the past two decades, scientists have consistently overestimated the ability of bio-technologies to compensate for a lack of knowledge and underestimated the complexity of the parasite and its interaction with its human host. Times have changed, and it is now more apparent that specific strategies aimed at reducing the parasite load or its clinical consequences should correspond to the diversity of clinical presentations and of immune responses to the parasite. This revival of interest in the 'natural history' of malaria is good news. The Newbold report demonstrates that welldesigned epidemiological studies that increase our understanding of the equilibrium between the parasite and its human host are necessary to improve malaria vaccine strategies.

1. Gupta, S., Snow, R.W., Donnelly, C.A., Marsh, K. \& Newbold, C. Immunity to severe malaria is acquired after one or two infections. Nature Med. 5, 340-343 (1999).

2. Trape, J.-F. et al. Impact of chloroquine resistance on malaria mortality. C.R. Acad. Sci. Paris. 321, 689-697 (1998).

3. Marsh, K. Malaria disaster in Africa. Lancet 352, 924 (1998).

4. Snow, R.W. et al. Relation between severe malaria morbidity in children and level of Plasmodium falciparum transmission in Africa. Lancet 349, 1650-1654 (1997).

5. Trape, J-F \& Rogier, C. Combating malaria morbidity and mortality by reducing transmission. Parasitol. Today 12, 236-240 (1996)

6. Druilhe, P., Sabchareon, A., Bouharoun-Tayoun, H., Oeuvray, C. \& Pérignon, J.-L. In vivo veritas : lessons from immunoglobulin-transfer experiments in malaria patients. Ann. Trop. Med. Parasitol. 91 (supp.1), S37-S53 (1997).

Bio-medical Parasitology

Institut Pasteur, 75015 Paris, France

\title{
NOS: Modulator, not mediator of cardiac performance
}

\begin{abstract}
The 'endothelial' isoform of nitric oxide synthase modulates (but is not obligatory for) cardiac muscle function; signaling by NO in different cellular compartments coordinates diverse effects within the cardiac myocyte (pages 331-334).
\end{abstract}

$T_{\mathrm{c} i \mathrm{n}}^{\mathrm{n}}$ HE MAMMALIAN HEART consists of two atrial chambers that collect blood and two ventricles that pump it. An electrical impulse initiated in the right atrium spreads through a conduction system to coordinate myocardial contraction (excitation-contraction coupling). Two isoforms of nitric oxide synthase (NOS), neuronal (nNOS or NOS1) and endothelial (eNOS or NOS3) - which catalyze the formation of nitric oxide (NO) and related signaling molecules from L-arginine-are variably distributed throughout the heart $^{1,2}$. The ventricular cardiac muscle cells (myocytes) contain both isoforms. NOS1 is expressed in the sarcoplasmic reticulum $^{1}$ (SR) and NOS3 in the sarcolemma and T-tubule membranes ${ }^{3}$ - sites notable for their control of calcium flux, which are the chief determinants of the force and rhythm of myocardial contraction (see Fig.). NOS3 is also found in the mitochondria ${ }^{4}$, and a third isoform, iNOS (NOS2), is expressed under pathophysiological conditions ${ }^{5}$.

Studies at the molecular, cellular and organismal levels have firmly established the influence of NO on cardiac performance, in particular, myocardial contractility $^{6,7}$, lusitropy ${ }^{8}$, chronotropy ${ }^{3}$, and energy production/consumption ${ }^{9}$. But how essential is NO to cardiovascular performance? On page 331 of this issue, Vandecasteele and

\section{JOSHUA M. HARE ${ }^{1} \&$ JONATHAN S. STAMLER ${ }^{2}$}

colleagues ${ }^{10}$ address the question in transgenic mice with a homozygous deletion in the gene encoding NOS3. They find no difference in muscarinic and $\beta$-adrenergic regulation of isolated myocyte heart rate, papillary muscle contractility, and calcium current in transgenic compared to wild-type mice. Their study is of particular interest because of the diametrically opposed results reported previously in the same animals by other groups ${ }^{3,11}$. As both sets of studies are well done, it would seem premature to conclude that the new data refute the previous findings.

Understanding how NO works helps to make sense of these differing observations. NO can signal indirectly through cyclic GMP, and, at least in the myocyte, this second messenger tends to offset the actions of cyclic AMP (see Fig.). In an alternate pathway, NO binds directly to cysteine sulfhydryls or iron centers in proteins (nitrosylation/redox signaling). The sarcolemmal L-type calcium channel ${ }^{12}$, the SR-calcium release channel ${ }^{13}$ (CRC) and the SR-calcium ATPase (ref.1) are all $\mathrm{NO} /$ redox-regulated. $\mathrm{NO}$ and related molecules also influence cellular respiration by binding to mitochondrial electron transport complexes and perhaps to aconitase and creatine kinase (see Fig.).

One compelling difference between the Vandecasteele study and the earlier studies is the age of the animals: 3-6 months in the former compared with 2 days $^{3}$ or 2-3 months in the latter (R. Gyurko \& P. Huang, personal communication). The evolution of compensatory mechanisms in transgenic mice is not reserved to embryogenesis, a fact brought to light by age-related changes in the blood pressure of animals with distal defects in the NOS/cGMP pathway ${ }^{14}$. Moreover, pathways that produce cGMP are redundant: heme oxygenases or atrial natriuretic peptides may compensate for NOS (ref. 15). Alternatively, nNOS might substitute for eNOS in the heart as it does in certain blood vessels ${ }^{16}$ (nNOS and iNOS activity were not measured by the Vandecasteele group). Inward acetylcholine-activated $\mathrm{K}^{+}$ channels in myocytes from NOS3-deficient mice that might contribute to muscarinic inhibition of the $\beta$-adrenergic $\mathrm{Ca}^{2+}$ current have also been described ${ }^{13}$.

Studies that probe NO function by inhibition of NOS - be it by pharmacologic or genetic means-can only reveal net effects. However, NO frequently exhibits dual activities. In particular, NO concentrations rise and fall in the heart on millisecond timescales ${ }^{17}$. Corresponding increases in cGMP inhibit contractility, 